\title{
Excitation of a Primordial Cold Asteroid Belt as an Outcome of Planetary Instability
}

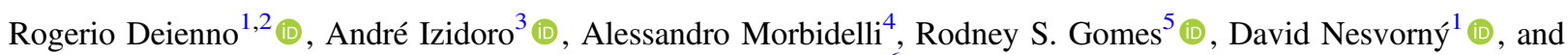 \\ Sean N. Raymond ${ }^{6}$ iD \\ ${ }^{1}$ Department of Space Studies, Southwest Research Institute, 1050 Walnut St., Boulder, CO 80302, USA; rdeienno@boulder.swri.edu \\ ${ }^{2}$ Instituto Nacional de Pesquisas Espaciais, Avenida dos Astronautas 1758, CEP 12227-010 São José dos Campos, SP, Brazil \\ ${ }^{3}$ Universidade Estadual Paulista, Grupo de Dinamica Orbital e Planetologia, Av. Dr. Ariberto Pereira da Cunha, 333, CEP 12516-410, Guaratinguetá, SP, Brazil \\ ${ }^{4}$ Laboratoire Lagrange, UMR7293, Université Côte d'Azur, CNRS, Observatoire de la Côte d'Azur, Boulevard de l'Observatoire, F-06304 Nice Cedex 4, France \\ ${ }^{5}$ Observatório Nacional, Rua General José Cristino 77, CEP 20921-400 Rio de Janeiro, RJ, Brazil \\ ${ }^{6}$ Laboratoire d'Astrophysique de Bordeaux, Univ. Bordeaux, CNRS, B18N, allée Geoffroy Saint-Hilaire, F-33615 Pessac, France \\ Received 2018 January 26; revised 2018 July 17; accepted 2018 July 19; published 2018 August 30
}

\begin{abstract}
The main asteroid belt (MB) is low in mass but dynamically excited. Here we propose a new mechanism to excite the MB during the giant planet (the "Nice model") instability, which is expected to feature repeated close encounters between Jupiter and one or more ice giants ("jumping Jupiter" or JJ). We show that, when Jupiter temporarily reaches a high-enough level of excitation, both in eccentricity and inclination, it induces strong forced vectors of eccentricity and inclination across the MB region. Because during the JJ instability Jupiter's orbit "jumps" around, the forced vectors keep changing both in magnitude and phase throughout the whole MB region. The entire cold primordial MB is thus excited as a natural outcome of the JJ instability. The level of such an excitation, however, is typically larger than the current orbital excitation observed in the MB. We show that the subsequent evolution of the solar system is capable of reshaping the resultant overexcited MB to its present-day orbital state, and that a strong mass depletion $(\sim 90 \%)$ is associated with the $\mathrm{JJ}$ instability phase and its subsequent evolution throughout the age of the solar system.
\end{abstract}

Key words: minor planets, asteroids: general - planets and satellites: dynamical evolution and stability

\section{Introduction}

The present-day asteroid main belt (MB) presents a challenge for theories of planet formation. Orbits within the MB have eccentricities ranging from 0 to $\sim 0.4$ and orbital inclinations from 0 to more than $20^{\circ}$. This high level of excitation is hard to reconcile with the presumably cold initial orbits of all planetesimals in the protoplanetary disk (including the MB asteroids and primordial trans-Neptunian objects)with eccentricities and inclinations near zero-as well as the cold orbits of the terrestrial and giant planets. The MB is low in mass, containing a total of just $\sim 5 \times 10^{-4} M_{\oplus}$ (DeMeo \& Carry 2013). This is far less (100-1000 times less) than the few Earth masses expected if the MB region were part of a disk with a smooth gradient in radial surface density (e.g., Hayashi 1981; Bitsch et al. 2015). Finally, the belt shows broad compositional diversity but is dominated by two prominent classes: the $\mathrm{S}$ types in the inner $\mathrm{MB}$ and $\mathrm{C}$ types in the outer $\mathrm{MB}$, albeit with significant overlap (Gradie \& Tedesco 1982; DeMeo \& Carry 2013, 2014). Addressing these constraints within a self-consistent framework of terrestrial and giant planet formation is an imposing theoretical challenge (e.g., Morbidelli et al. 2007; Hansen 2009; Walsh et al. 2011; Izidoro et al. 2015; Levison et al. 2015a, 2015b; Walsh \& Levison 2016; Raymond \& Izidoro 2017a, 2017b).

Some theories succeeded in matching the MB's level of excitation but presented other major problems. The "classical model" of terrestrial planet formation includes a distribution of planetary embryos extending out into the asteroid belt (Chambers \& Wetherill 1998; Chambers 2001; O'Brien et al. 2006; Raymond et al. 2006), which naturally excited the surviving asteroids (Chambers \& Wetherill 2001; Petit et al. 2001; O'Brien et al. 2007). However, the classical model has a well-known Achilles heel: it systematically results in the formation of Mars analogs almost as massive as Earth (Wetherill 1991; Raymond et al. 2009; Morishima et al. 2010) and very often leaves planetary embryos surviving in the belt, which is not consistent with the current observations of the MB (Raymond et al. 2009).

Sweeping secular resonances during planetesimal-driven migration of Jupiter and Saturn were also proposed to excite the MB (Minton \& Malhotra 2011; Lykawka \& Ito 2013). However, this model requires a fast migration for Saturn ( $\dot{a} \sim 4 \mathrm{au} \mathrm{Myr}^{-1}$ in the case of an initially cold $\mathrm{MB}$ or $\dot{a} \sim 0.8 \mathrm{au} \mathrm{Myr}^{-1}$ for an initially hot MB) with Jupiter fixed at $\sim 5.2$ au (Minton \& Malhotra 2011). According to Morbidelli et al. (2010), a more realistic timescale of migration for Jupiter and Saturn embedded in a planetesimal disk should be $\tau \sim 5$ Myr. Planet migration on this timescale would result in an $\mathrm{MB}$ incompatible with that currently observed, where one would obtain an inner belt with a larger fractional number of asteroids in highly inclined orbits than is observed today (Morbidelli et al. 2010; Walsh \& Morbidelli 2011; Toliou et al. 2016).

Rather than a smooth giant planet migration, an early "Nice model" planetary instability has the potential to explain the asteroid belt's orbital structure. In the Nice model, the giant planets formed in a more compact and more circular and coplanar configuration than their current one, and they achieved their current configuration after a phase of dynamical instability after gas dispersal. Clement et al. (2018) showed that the instability can produce sufficient excitation and mass depletion exterior to $\sim 1.5$ au to explain the small mass of Mars. While promising in terms of solving the small-Mars problem, the simulations of Clement et al. (2018) did not have highenough resolution to fully populate the MB. Rather, while they did provide a decent match to the $\mathrm{MB}$, they were forced to 
coadd many simulations to produce a model belt (Figure 6 in Clement et al. 2018).

The Grand Tack model (GT; Walsh et al. 2011) was the first model to match the inner solar system in a single evolutionary scenario. In the GT, Jupiter is assumed to have formed beyond the snow line and migrated inward via planet-gas disk interactions (e.g., Kley \& Nelson 2012; Baruteau et al. 2014). Meanwhile, Saturn grew and migrated inward toward Jupiter (Masset \& Papaloizou 2003). When Saturn caught up with Jupiter, the planets became locked in either mutual $3: 2$ or $2: 1$ mean motion resonance (MMR; Masset \& Snellgrove 2001; Morbidelli \& Crida 2007; Pierens \& Nelson 2008; Pierens \& Raymond 2011; Pierens et al. 2014). At this point, the planets' directions of migration were reversed (this happened when Jupiter was at around 1.5-2 au; Brasser et al. 2016), and both planets migrated outward until the gas in the disk dissipated, reaching their preinstability location (Nesvorný \& Morbidelli 2012; Deienno et al. 2017).

Within the framework of the GT model, Jupiter's and Saturn's excursion into the terrestrial and MB region has several implications. It confines the distribution of most solid material within $\sim 1$ au from the Sun, explaining why Mars accretion stopped and the planet remained small (Wetherill 1978; Hansen 2009). The excursion of Jupiter through the asteroid belt can also explain how the S-type and C-type asteroids were implanted into the $\mathrm{MB}$ region, with $\mathrm{S}$ types originating interior to Jupiter's original orbit and the $\mathrm{C}$ types farther out (Walsh et al. 2012). The resulting MB population is dynamically excited, the two asteroid types are partially mixed, and the total mass is just a few times the current one. In fact, the MB after the GT is overexcited compared with the present-day belt, but Deienno et al. (2016) showed that the subsequent $\sim 4.5$ Gyr evolution of the solar system naturally erodes the overexcited component so that the final distribution matches the present-day MB. A caveat, however, is that the inclination distribution out of the GT simulations should be confined within $\sim 20^{\circ}$ in order to reproduce the current ratio in the number of asteroids above and below the $\nu_{6}$ secular resonance.

Despite the success of the GT model, the scenario remains controversial (see Raymond \& Morbidelli 2014 for a critical review). The key uncertainty is related to the outward migration mechanism of the planets, which has not been validated when gas accretion onto the giant planets is taken into account in a self-consistent way (D'Angelo \& Marzari 2012). Indeed, in an isothermal disk with Jupiter and Saturn in 3:2 MMR, the planets only migrate outward for Jupiter-to-Saturn mass ratios between roughly 2 and 4 (Morbidelli \& Crida 2007). Of course, this ratio was evolving while the planets were accreting gas, with a direct feedback between the giant planets' growth and migration.

Another class of models posit that the asteroid belt had a low mass from the very beginning (Izidoro et al. 2015; Levison et al. 2015b; Moriarty \& Fischer 2015; Ogihara et al. 2015; Drążkowska et al. 2016). These models argue that the drift of small particles due to aerodynamics drag could have concentrated material near $1 \mathrm{au}$, leaving little mass in the asteroid belt. Starting from a steep-enough radial surface density distribution of solid material, Izidoro et al. (2015) was indeed able to build terrestrial planets similar to the real one, with a large Earth/ Mars mass ratio, but they could not explain the orbital excitation of the MB (which remained too dynamically cold) nor its taxonomical mixture. However, additional mechanisms have been proposed that could reconcile the low-mass asteroid belt model with the present-day $\mathrm{MB}$ and provide a viable alternative to the GT model. Raymond \& Izidoro (2017a, 2017 b) showed that even if the MB was originally empty or almost empty (compatible with the low-mass asteroid belt scenario), the growth of Jupiter and Saturn during the gas disk phase naturally implants scattered primordial planetesimals into the MB region. Planetesimals from the Jupiter-Saturn region and beyond are scattered inward during the giant planets' growth and implanted into the belt under the action of aerodynamic gas drag (Raymond \& Izidoro 2017a). They could correspond to the C-type asteroids that we observe today. In addition, planetesimals scattered outward from the terrestrial planet-forming region, due to their interaction with rogue planetary embryos, can be implanted onto main belt orbits by resonant interactions with Jupiter (Bottke et al. 2006; Raymond \& Izidoro 2017b). They could correspond to the S-type asteroids.

While this may solve the problem of the MB's taxonomical mixture, a problem persists. The dynamical state of the MB is still cold, mainly because the implantation of C-type asteroids occurs via gas-drag damping, such that all but the largest ones $(D=1000 \mathrm{~km})$ end up with orbital eccentricity and inclinations near zero (see Figure 3 in Raymond \& Izidoro 2017a). One proposed solution is the chaotic excitation model described by Izidoro et al. (2016). In this model, Jupiter and Saturn are initially in MMR (as predicted by migration models) but are not very close to the resonance center, so they have some chaotic motion on secular timescales. It remains to be demonstrated whether such a specific configuration is consistent with migration models of resonant capture. Indeed, the chaotic excitation has only been demonstrated when Jupiter and Saturn are initially locked in their mutual 2:1 MMR and for specific configurations inside this resonance. However, according to Nesvorný \& Morbidelli (2012) and Deienno et al. (2017), it is more likely to reconstruct the orbital architecture of the outer solar system with a giant planet instability if Jupiter and Saturn were initially in the 3:2 MMR.

The goal of this paper is to better understand the evolution and dynamical excitation of a cold primordial MB during the giant planet dynamical instability. Our study starts from a best guess for the initial configuration of the giant planets proposed by Deienno et al. (2017), ${ }^{7}$ with Jupiter and Saturn initially locked in their 3:2 MMR. We show how the evolution of Jupiter is the key to understanding both the excitation of the MB and the chaotic evolution by Izidoro et al. (2016), when starting from a resonant configuration of the giant planets. Our approach is similar to that of Clement et al. (2018) but with two main differences. First, we start with a low-mass asteroid belt with the goal of demonstrating dynamical excitation with little focus on mass depletion. Second, we consider enough particles in order to assess the final orbital distribution in the asteroid belt with good statistics.

This study is not meant to disprove either the GT or the chaotic excitation model. Rather, we describe a new mechanism for exciting the asteroid belt starting from a dynamically cold, low-mass setup. Our mechanism is a by-product of the giant planet instability, and, even though the belt may be

\footnotetext{
Similar results could be expected from the evolution proposed by Gomes et al. (2018), because what matters is the evolution of Jupiter during the JJ instability phase and not the one of Neptune as in Nesvorný (2015).
} 
temporarily overexcited, subsequent dynamical evolution brings it to a state consistent with the present-day belt. It is also outside the scope of the present paper to try to provide an exact match to the present-day MB, which would demand a prohibitive number of simulations and testing over too large a number of parameters.

Our paper is structured as follows. In Section 2 we present our instability model and the effects that it has on a primordial cold MB. In Section 3 we discuss the direct effect that each planet has upon the MB. Section 4 is devoted to understanding the mechanism of excitation working behind our results. In Section 5 we discuss the implications of our results for the time of the planetary instability, by considering the effects on the excitation of terrestrial planets. We compare the MB from our simulations with the present-day asteroid main belt in Section 6, where we also discuss the constraint on the initial mass of the asteroid belt. Lastly, Section 7 concludes the paper.

\section{Planetary Instability and the Excitation of the MB}

We designed a set of simulations to test whether the MB could have been excited by the giant planet instability.

We first performed simulations of the instability with the goal of finding cases that matched all of the constraints listed in Nesvorný \& Morbidelli (2012) and Deienno et al. (2017). Simulations started from the initial configuration of a system with five giant planets proposed by Deienno et al. (2017), with $a_{\mathrm{J}} \sim 5.4 \mathrm{au}$ and more distant planets locked in a resonant chain, with MMRs between adjacent planets of 3:2, 3:2, $2: 1$, and 3:2. Uranus, Neptune, and the additional planet are assumed to have a mass of $\sim 15$ Earth masses, and Jupiter and Saturn have their current masses. In this configuration, all planets were initially in quasi-circular and planar orbits, as predicted by hydrodynamical simulations of planet migration that explain the formation of the aforementioned resonant chain (Morbidelli et al. 2007). We assume the existence of a planetesimal disk composed of 35 Earth masses equally divided within 1000 planetesimals with zero eccentricity and inclinations ranging from $0^{\circ}$ to $1^{\circ}$. The planetesimal disk was designed to have a surface density of $1 / r$, and the inner and outer edges were set to be 21 au and $30 \mathrm{au}$, respectively.

We performed over 1000 simulations leading to planetary instability of the jumping-Jupiter (JJ) kind. These are evolutionary pathways in which Jupiter is involved in close encounters with another planet, typically a Neptune-mass planet that is ejected from the system. As a consequence of these encounters, the orbital distance between Jupiter and Saturn jumps abruptly (hence the name "JJ"). We extended most simulations to $10 \mathrm{Myr}$ after the instability but could not control the exact timing of the instability. The integrations were performed using the Mercury (Chambers 1999) hybrid integrator with a time step of $0.5 \mathrm{yr}$. The outer-disk planetesimals perturbed the planets' orbits but did not selfinteract.

Figure 1 shows the most successful simulation from this batch. It matches all of the constraints presented in Nesvorný \& Morbidelli (2012) and Deienno et al. (2017):

(A) The final planetary system has four giant planets.

(B) The final semimajor axis of each planet is within $20 \%$ of its current value (here we got all planets within 5\%), and the final mean eccentricities and inclinations are no larger than $0^{\circ} .11$ and $2^{\circ}$, respectively.

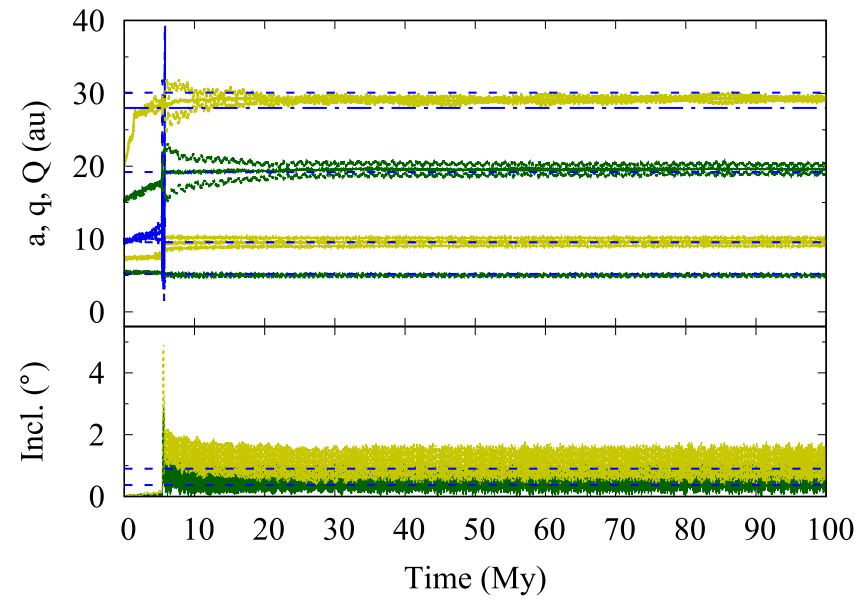

Figure 1. Top: evolution of the semimajor axis, perihelion, and aphelion of Jupiter, Saturn, and three ice giant planets. The initial resonant configuration of the planets was $3: 2,3: 2,2: 1$, and $3: 2$ with Jupiter at $a_{\mathrm{J}} \sim 5.4$ au. Bottom: evolution of the inclination of Jupiter (green) and Saturn (yellow). The dashed lines in the top panel represent the current semimajor axes for the four solar system giant planets, and the dot-dashed line demarcates 28 au, where Neptune likely needs to have been at the onset of the planetary instability (Nesvorný, 2015 ) in order to reproduce the so-called kernel of the Kuiper Belt (Petit et al. 2011). In the bottom panel, the dashed lines represent the current mean inclination for Jupiter and Saturn with respect to the invariable plane (the plane perpendicular to the total angular momentum of the solar system excluding the recently proposed planet 9; Batygin \& Brown 2016), from Nesvorný \& Morbidelli (2012), 0.37 and 0.90 , respectively.

(C) The proper mode of Jupiter's eccentricity $e_{55}$ is at least half of its current value of 0.044 (see Figure 3 for (C) and (D)).

(D) The ratio between the orbital period of Jupiter and Saturn $\left(P_{\mathrm{S}} / P_{\mathrm{J}}\right)$ evolves from $<2.1$ to $>2.3$ in less than $1 \mathrm{Myr}$.

(E) Neptune migrates ahead of all other planets such that the planetary instability happens when 27 au $<a_{N}<29$ au, namely 28 au (Nesvorný 2015).

Criterion (D) is generally invoked to guarantee the stability of the terrestrial planets in the case of a late giant planet instability in the solar system (Gomes et al. 2005; Brasser et al. 2009; Agnor \& Lin 2012; Bottke et al. 2012). However, it is important even in the case of an early (preterrestrial planet formation) instability because criterion (D) guarantees that the inner main belt is not overexcited in inclination (Walsh et al. 2011; Toliou et al. 2016).

Criterion (E) is imposed to make the planet evolution consistent with the current structure of the Kuiper Belt (Nesvorný 2015).

The simulation from Figure 1 represents a self-consistent evolution of the solar system, and we refer to it as the nominal simulation. ${ }^{8}$

Next, we "embedded" a primordial cold asteroid belt into this nominal simulation. To save CPU time, we resimulated our nominal case and recorded the orbits of Jupiter, Saturn, and the

\footnotetext{
8 We did not restrict ourselves to only this simulation. Several other cases confirm the findings we will report with the nominal simulation. However, most of these additional cases do not satisfy all constraints (A)-(E). In fact, finding a case like the one in Figure 1, which satisfies all constraints and still accounts for the evolution that we will discuss throughout the paper, in a small number of attempts is really difficult, given the large variety of evolutions resulting from an instability simulation. Because of that, to shorten the discussion, we decided to focus on only this case. Therefore, although the results we will present may not be achieved for every instability, they certainly will every time that the instability happens as reported here.
} 

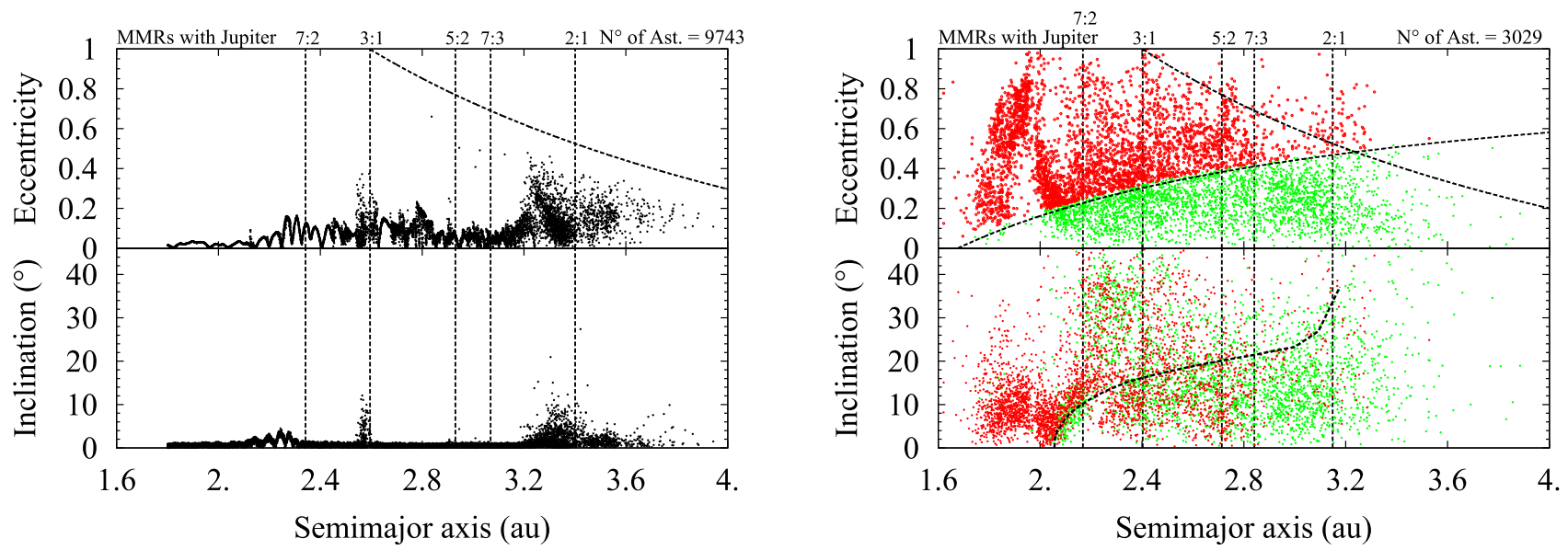

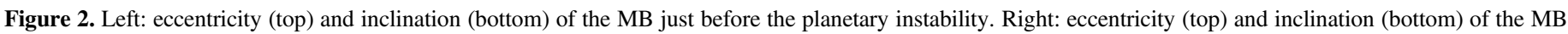

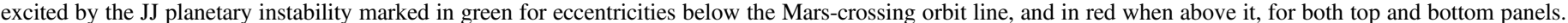

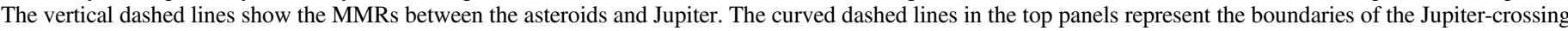

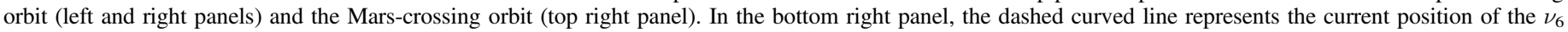
secular resonance.

ejected extra ice giant (hereafter $p l 5$ ) at every 1 yr output, starting from $\sim 2.5$ Myr before the planetary instability for a total integration time of $5 \mathrm{Myr}$. From these outputs, we interpolated the orbits of the planets (using linear interpolation of their orbital elements) from $\sim 2.5 \mathrm{Myr}$ before to $\sim 2.5 \mathrm{Myr}$ after the planetary instability. The orbits of Uranus, Neptune, and of the trans-Neptunian planetesimals were not considered (but their presence is indirectly recorded in the orbital evolution of the considered planets). We included a cold MB extending from 1.8 to 3.6 au consisting of 10,000 massless particles with eccentricities equal to zero and inclinations ranging from $0^{\circ}$ to $1^{\circ}$. The other angular elements were drawn randomly within the interval $0^{\circ}-360^{\circ}$. We modified the Mercury integrator to include the planet's interpolated orbits in order to compute the external force that perturbs the test particles, and we reduced the integration time step to 10 days. The terrestrial planets were also ignored in this phase of the simulation. Although this may prevent most of the high-eccentricity asteroids in the inner MB from being removed, at this point we are only interested in evaluating the level of excitation provided by the instability of the giant planets. Having the terrestrial planets included in the simulation would probably cause some depletion of the asteroids colored in red in Figure 2 (right panels). However, the terrestrial planets would not significantly modify the precession rates of the MB asteroids, which are dominated by Jupiter (see Equation (7.55) of Murray \& Dermott 1999) and, as we show below, is the main driver of the excitation process. Therefore, including the terrestrial planets is not important during the instability. Not knowing whether or not the terrestrial planets already existed at that time, we prefer not to include them. Of course, they will be included in the subsequent simulation on the long-term evolution of the MB in the postexcitation phase.

The MB's final level of excitation from this nominal simulation is shown in the Figure 2 right panel, and the state of the MB just before the $\mathrm{JJ}$ instability is shown in the Figure 2 left panel. The excitation is similar to those presented in Deienno et al. (2016) for the GT and in Izidoro et al. (2016) for the chaotic excitation and greatly exceeds the current dynamical excitation of the MB. This suggests that, whatever the excitation mechanism, an overexcitation phase for the MB seems unavoidable. However, a close look at the evolution of the $\mathrm{MB}^{9}$ showed no path to orbital chaos prior to the instability when the initial configuration of the planets was $3: 2,3: 2,2: 1$, and $3: 2$. In other words, as there was no chaotic excitation before the planetary instability, unlike in Izidoro et al. (2016), a different mechanism is at play. Still, a very strong perturbation occurred during a very short window of time $(<200 \mathrm{kyr})$ within the JJ instability period ( $\sim 800 \mathrm{kyr}$ or less; see Figure 3$)$.

As a last note, it is important to say that, although Jupiter and Saturn go beyond the 5:2 MMR (i.e., their final separation is larger than their current one), the crossing of the 5:2 MMR seems to play no important role in the entire excitation process. Thus, the results should not be an artifact of this feature in the planets' evolution.

\section{The Role of Each Planet}

To better understand the MB excitation during the planetary instability, we now focus on the short window of time (800 kyr) when the instability happens. Figure 3 shows the evolution of the eccentricity and inclination of Jupiter (green), Saturn (yellow), and pl5 (blue) during that window of time, as well as the evolution of $P_{\mathrm{S}} / P_{\mathrm{J}}$ (green) and of the perihelion of $p l 5$ $\left(q_{p l 5}\right.$, blue). Figure 3 shows the interval before and after the instability shown in Figure 1 in much more detail.

Our next step was to test the individual effects of Jupiter, Saturn, and pl5 on MB excitation. We used the orbits of Jupiter, Saturn, and pl5 interpolated during the $800 \mathrm{kyr}$ of Figure 3 to simulate their individual effects on an initially cold MB. The initial belt was the same as previously described, with 10,000 massless particles with $a=[1.8-3.6]$ au, $e=0$, incl. $=[0-1]^{\circ}$, and $\omega, \Omega, l=[0-360]^{\circ}$. We effectively reran the same MB three times but considered only one of the three planets in each simulation. As the orbits of the planets were interpolated, we did not lose any detail in the path followed by each of them. Of course the frequencies that they induce in the

\footnotetext{
9 An animation of the entire evolution can be found electronically at http:/ staff.on.br/rodney/rogerio/mb-excitation.mp4 with Jupiter (gray), Saturn (orange), and $\mathrm{pl5}$ (green)
} 


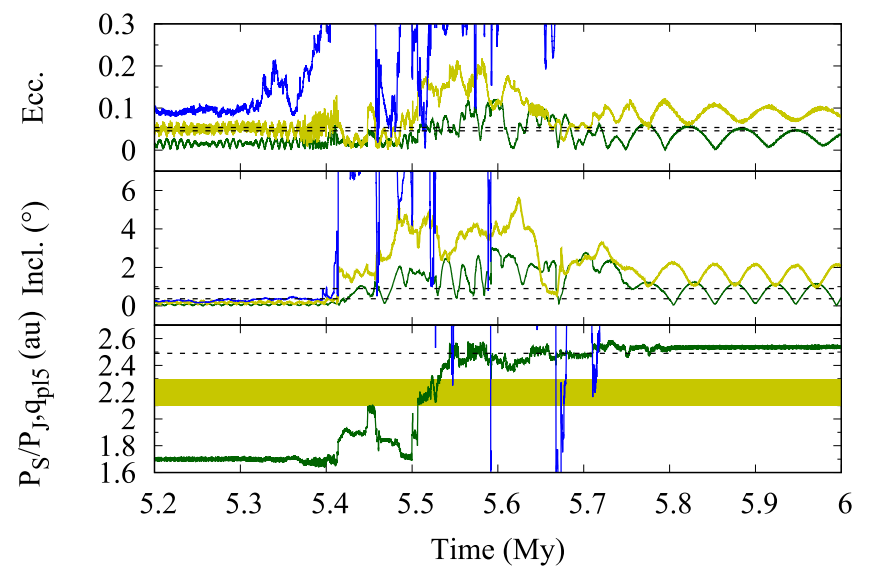

Figure 3. Evolution of the eccentricity (top) and inclination (middle) for Jupiter (green), Saturn (yellow), and pl5 (blue); bottom panel: the evolution of $P_{\mathrm{S}} / P_{\mathrm{J}}$ (green) and of perihelion of $p l 5\left(q_{p l 5}\right.$, blue). The horizontal dashed lines in the top and middle panels represent the present-day mean values of Jupiter and Saturn eccentricities and inclinations, respectively. The yellow shaded area in the bottom panel represents $2.1<P_{\mathrm{S}} / P_{\mathrm{J}}<2.3$, where the period ratio has to spend the shortest possible time in order to avoid a strong depletion of the inner asteroid belt (Morbidelli et al. 2010), and the horizontal dashed line in the same panel shows the current value of $P_{\mathrm{S}} / P_{\mathrm{J}}$.

MB change by changing the number of disturbing planets, but this experiment was simply to evaluate only the direct effect caused by each planet.

Figure 4 shows the eccentricity and inclination distributions for main belt asteroids at the end of the $800 \mathrm{kyr}$ simulation passing through the JJ planetary instability when considering only Jupiter (left), only Saturn (center), and only pl5 (right).

It is clear from Figure 4 that Jupiter is the planet responsible for exciting the MB during the planetary instability. The nonrandomized eccentricity and inclination excitation between $2 \mathrm{au}$ and $2.4 \mathrm{au}$ in the left panel of Figure 4 is most probably related to the incorrect secular frequencies of asteroids due to the absence of the direct effect of Saturn, which displaces the location of secular resonances. Even so, the result itself is very similar to that presented in the Figure 2 right panel where all three planets (Jupiter, Saturn, and pl5) were considered together, especially when considering the green-colored asteroids below the Mars-crossing line. Saturn alone (middle panels of Figure 4) seems to have an almost negligible direct effect upon a primordially cold MB. Surprisingly, even $p l 5$, which has a high eccentricity and a perihelion distance well inside the $\mathrm{MB}$ for some periods during the considered time interval $\left(q_{\min } \sim 0.57 \mathrm{au}\right.$ at $t \sim 5.59 \mathrm{Myr}$, when $a \sim 3.15 \mathrm{au}$, $e \sim 0.82$, and $i \sim 9.37$ or $q_{\min } \sim 1.26$ au at $t \sim 5.67 \mathrm{Myr}$, when $a \sim 5.34$ au, $e \sim 0.76$, and $i \sim 11.66$; Figure 3 bottom panel), does not excite the initially cold MB to the level in the Figure 2 right panel. Most probably, the weakness of $p l 5$ in exciting the $\mathrm{MB}$ is related to its large eccentricity and inclination when attaining a low pericenter distance. Only the regions of the MB with $a>2.8$ au faced some nonnegligible disturbance, but the eccentricities reached no more than 0.4 and inclinations barely reached $10^{\circ}$ (Figure 4 right panel). The question that remains is, why is Jupiter alone causing so much excitation, mainly in inclination? The answer can likely offer a complementary explanation of the chaotic evolution seen in Izidoro et al. (2016) and can also be related to the dispersal of asteroidal families during the planetary instability proposed by
Brasil et al. (2016), although with somewhat smaller dispersion in the inclinations in their work.

In fact, the excitation of the inclination is difficult to understand. The Jupiter-Saturn system has only one secular frequency, called $s_{6}$, associated with the motion of their longitudes of the node. So, there is only one nodal secular resonance possible between the asteroid and the planets: $s=s_{6}$, also known as $\nu_{16}$. In the JJ, the $\nu_{16}$ resonance jumps across most of the asteroid belt, instead of sweeping through it, so one should expect that the middle and outer parts of the asteroid belt remain unexcited in inclination.

\section{Mechanism of Excitation}

Having in mind that the inclination excitation of the MB is the key to constraining the evolution of Jupiter during the JJ phase, we closely studied the inclination evolution of selected MB test particles. We randomly choose a few MB test particles across the MB range. What we found is that the response in the inclination of all selected $\mathrm{MB}$ test particles was related to changes in the inclination of Jupiter. Every time the inclination of Jupiter went up or down, the inclination of the MB test particles responded by going up or down and also acquiring different amplitudes of oscillations. Such a response might be an effect of the forced inclination of Jupiter acting upon all MB test particles.

Figure 5 illustrates this phenomenon. It plots $I \sin \left(\Omega-\Omega_{\mathrm{J}}\right)$ versus $I \cos \left(\Omega-\Omega_{\mathrm{J}}\right)$ for four MB test particles during three different epochs: before, during, and after the planetary instability.

The different behaviors shown in these plots indicate the following: (1) In the beginning, when Jupiter has a wellbehaved quasi-circular and planar orbit, the forced inclination vector $\left(I_{\text {forced }}\right)$ felt by the MB test particles is small (small central black circle in the panels of Figure 5). (2) At the onset of the instability, when Jupiter gets some inclination, it induces an instantaneous, large forced inclination vector on the MB test particles. Then, as Jupiter jumps around, the magnitude and phase of the $I_{\text {forced }}$ vector change. Thus, at different locations within the $M B$, different $M B$ test particles, with different longitude of ascending node, can undergo different amplitudes of oscillations. For this reason, the final inclination excitation is not uniform (i.e., with all particles within a given semimajor axis reaching the same inclination). Instead, particle inclinations are randomized, filling all of the inclination range (red curve in the panels of Figure 5). (3) Once the planetary instability phase ends and Jupiter returns to a more wellbehaved, quasi-circular, and low inclined orbit, ${ }^{10}$ the MB test particles return to face a constant magnitude of the inclination forced vector, and because of that, their proper inclinations are frozen at the values acquired during the $\mathrm{JJ}$ instability phase (blue curves in the panels of Figure 5).

This mechanism is consistent with the chaotic excitation of Izidoro et al. (2016), although in that model this happened during the phase when Jupiter and Saturn were in their mutual 2:1 MMR, whereas here it occurs when the planets are evolving in semimajor axis under the effects of mutual close encounters. Our results therefore suggest a self-consistent evolution that

\footnotetext{
${ }^{10}$ Although in the end of the $800 \mathrm{kyr}$ simulation (Figure 3 middle panel), Jupiter's inclination is higher than its current value and with large oscillations, the Figure 1 bottom panel shows that such inclination will continue damping over the next $100 \mathrm{Myr}$, matching perfectly the constraints imposed and well representing Jupiter's current inclination.
} 

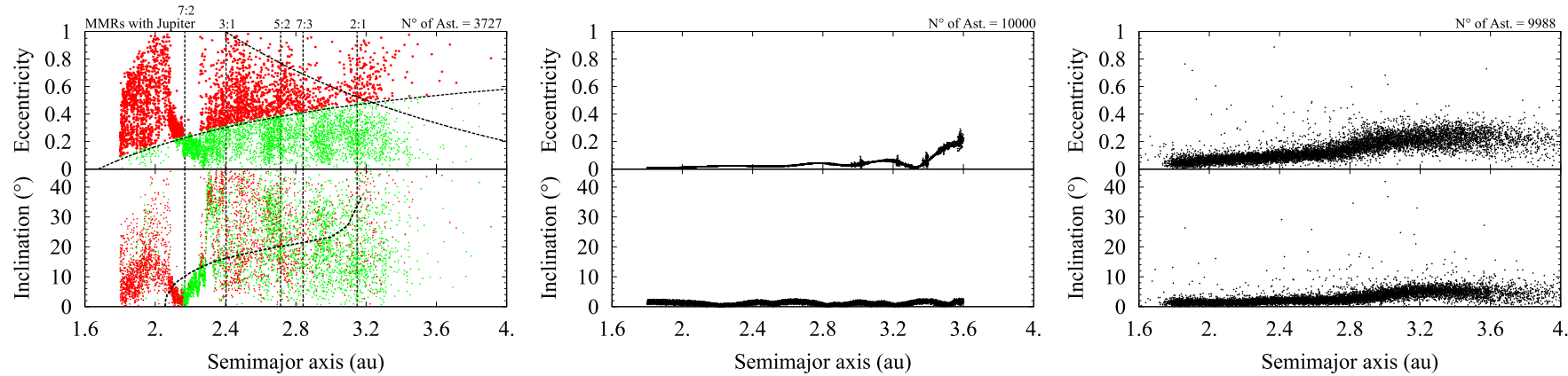

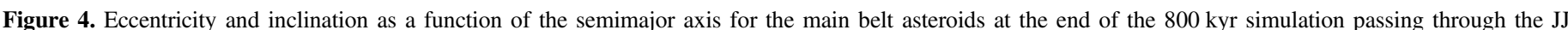

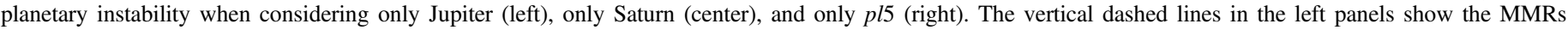

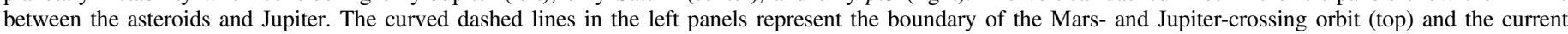

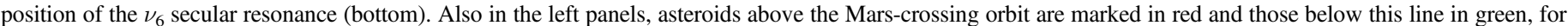
both top and bottom panels.
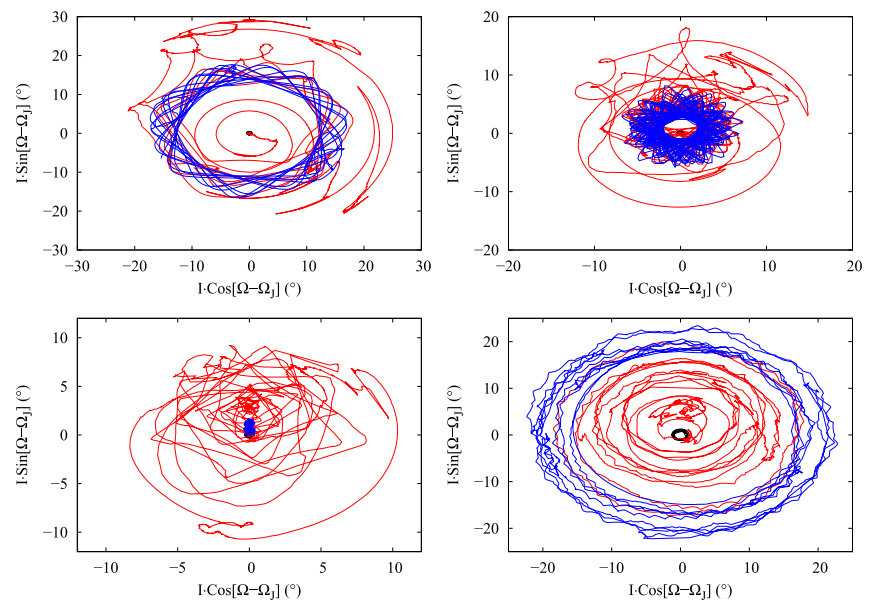

Figure 5. $I \sin \left(\Omega-\Omega_{\mathrm{J}}\right)$ vs. $I \cos \left(\Omega-\Omega_{\mathrm{J}}\right)$ for four randomly selected $\mathrm{MB}$ test particles, for three different epochs, before (small central black circle), during (red), and after (blue) the planetary instability.

leads to a pathway for a possibly chaotic excitation of the MB induced by the JJ planetary instability, which works also with Jupiter and Saturn initially in their 3:2 MMR.

Summarizing, to obtain a well-excited MB from a primordially cold one, all orbital elements of Jupiter have to change several times during the JJ instability phase. Jupiter must also acquire a nonnegligible inclination during the instability phase to excite the MB inclinations. Although in our runs that led to a high-enough level of inclination excitation of the MB the orbital inclination of Jupiter was $\sim 1^{\circ} .5$ to $\sim 3^{\circ}$ during the $\mathrm{JJ}$ instability (Figure 3), we cannot at this point constrain the minimum inclination that Jupiter had to achieve. This is because the forced inclination vector faced by the MB test particles does not depend directly on Jupiter's inclination, but rather on the amplitude of the $s_{6}$ frequency in its secular motion, the value of the latter, and the frequencies and associated amplitudes induced by the presence of the ice giant planets. This becomes evident when developing an analytic theory for secular motion, along the lines of Chapter 7 in Murray \& Dermott (1999). Because in the JJ evolution the orbits of the planets keep changing, the frequencies, the secular phases, and the amplitudes keep changing as well.

Finally, it is also important to discuss three other aspects:

(i) As the instability depicted in this work requires a relatively long sequence of Jupiter's jumps, it is important to check that a long phase of close encounters between Jupiter and $p l 5$ does not destabilize the regular satellite system of Jupiter (Deienno et al. 2014). Deienno et al. (2014) concluded that what is important for the stability of the satellites is not the total number of encounters, but the distance to the closet one. Following Deienno et al. (2014), the orbits of the Galilean satellites can be profoundly affected if the encounter distance $\left(d_{\text {enc }}\right)$ between Jupiter and $p l 5$ is $d_{\text {enc }}<0.03 \mathrm{au}$. They found that $0.03 \mathrm{au}<d_{\text {enc }}<0.05$ au causes only small variations in the orbital elements of the Galilean satellites, and that $d_{\text {enc }}>0.05$ au leaves the satellite system essentially undisturbed. In our nominal simulation, we never get $d_{\text {enc }}<0.05$ au. To be more precise, the three closest encounters we measured were $d_{\text {enc }} \sim 0.05$ au at $\mathrm{t} \sim$ $5.46 \mathrm{Myr}, d_{\mathrm{enc}} \sim 0.06 \mathrm{au}$ at $\mathrm{t} \sim 5.59 \mathrm{Myr}$, and $d_{\mathrm{enc}} \sim$ 0.08 au at $\mathrm{t} \sim 5.60 \mathrm{Myr}$. Therefore, our simulated evolution should not put at risk the orbital structure of the Galilean satellites.

(ii) Although very similar, this work presents some differences from those of Morbidelli et al. (2010) and Brasil et al. (2016) for what concerns the excitation achieved for the orbital inclination of the MB. The main difference between these works is that we have a high time-resolved orbital evolution for Jupiter, which is also involved in a much larger series of encounters (a case well preferred by Nesvorný et al. 2014 for the capture of irregular satellites). This leads Jupiter to change a lot in $a, e, i$, and with that the forced inclination vector. This difference alone is enough to make this case different from Morbidelli et al. (2010), which, without pl5, could not have this richness in stochastic events in Jupiter's evolution. In our understanding, the fact that Jupiter acquires some $\sim 1.5$ to $\sim 3^{\circ}$ inclination when receiving several semimajor axis kicks during the instability phase is what makes the results in this work different from those of Brasil et al. (2016). Recall that, although considering only families and not the entire MB, similar to this work, Brasil et al. (2016) claim that the dispersion of the primordial families beyond recognition due to the planetary perturbations occurs particularly in inclination. However, like Morbidelli et al. (2010), they pointed out that the dispersion in inclination decreases with the increase in the semimajor axis within the MB. We argue that, with a more jumpy orbital evolution for Jupiter, acquiring a higher excitation in orbital inclination, Brasil et al. (2016) would find results 
similar to those presented here. Therefore, Brasil et al. (2016) and this work show that both a strong and a weak inclination excitation of the MB are possible, depending on the exact evolution of Jupiter's orbit.

(iii) Until now, we only looked at the influence of the JJ instability on the $\mathrm{MB}$, from $\sim 1.8 \mathrm{au}$ to $\sim 3.6 \mathrm{au}$. However, it is possible that some large values of forced eccentricity and inclination vectors influence the terrestrial planet region as well. The next section will address this issue.

\section{Implication for the Time of the Planetary Instability}

Here we address the dynamical excitation of terrestrial bodies during our nominal JJ planetary instability.

We once again make use of the $800 \mathrm{kyr}$ instability phase of our nominal simulation shown in Figure 3. We consider three different situations:

1. An early instability scenario with 10,000 massless test particles spread from $0.5 \mathrm{au}$ to $4 \mathrm{au}$, with $e=0$, Incl. $=[0-1]^{\circ}$, and $\omega, \Omega, l=[0-360]^{\circ}$.

2. A late instability scenario, with no MB test particles but including the fully formed Venus, Earth, and Mars. In this case, we are only interested in how the terrestrial planets would respond to the JJ planetary instability and do not address the excitation of the MB because it was done in the previous sections. We consider all planets initially with their current orbits, with inclinations referred to the invariable plane (Souami \& Souchay 2012), as defined in the caption of Figure 1.

3. A late instability scenario as in (2) but assuming all terrestrial planets from Venus to Mars have originally circular and planar orbits.

By looking at the evolution in the terrestrial planet region, our goal is to address whether the planetary instability is more likely to have happened early (Kaib \& Chambers 2016; Nesvorný et al. 2017; Clement et al. 2018; Morbidelli et al. 2018) or late (Gomes et al. 2005; Bottke et al. 2012; Brasser et al. 2013; Roig et al. 2016) in the evolution of the solar system.

In other to account for the perturbations of Jupiter, Saturn, and pl5 on Venus, Earth, and Mars, we updated our interpolation code of Section 2 so that terrestrial planets could also feel the external interpolated disturbers. So, for (2) and (3) above, all terrestrial planets interact with each other and are perturbed by Jupiter, Saturn, and $p l 5$. As for (1), we continue using the code described in Section 2, where test particles do not interact among themselves and only feel the perturbation of the interpolated planets.

Figure 6 (top panels) shows the outcome of experiment (1) after $800 \mathrm{kyr}$. The overall level of excitation in eccentricity and inclination as a response to the $\mathrm{JJ}$ instability appears to be much less effective in the terrestrial planet region. Although Figure 6 (top panels) presents some nonnegligible values $(\sim 0.2$ of eccentricity between 1 and $1.5 \mathrm{au}$ ), the overall result is consistent with the findings described by Clement et al. (2018), where the region within $a<1.8$ au was much less disturbed than the region for $a>1.8 \mathrm{au}$.

Figure 6 middle and bottom panels, on the other hand, show a very different behavior for the already-formed terrestrial planets. The evolution of Venus (yellow), Earth (blue), and
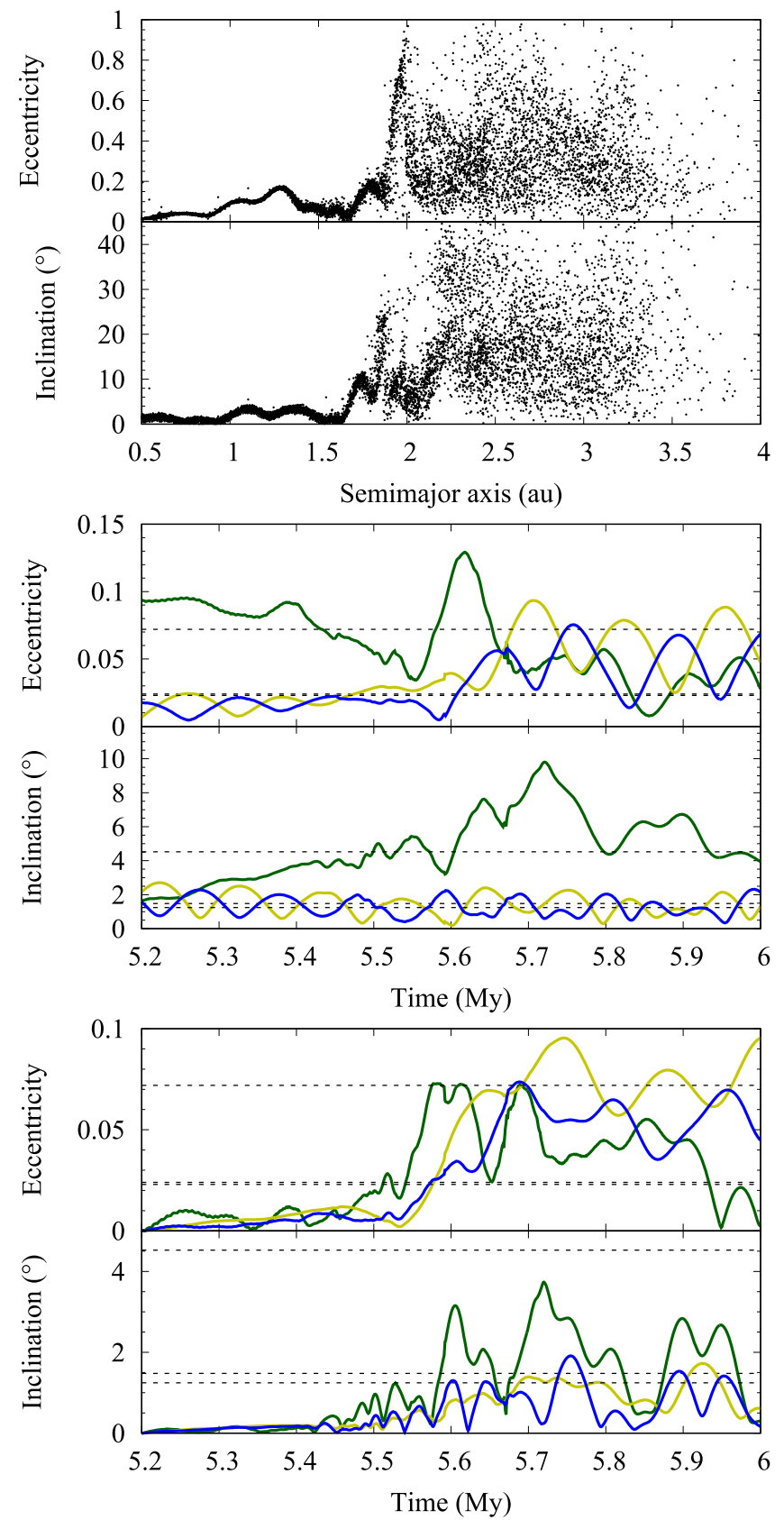

Figure 6. Top: distribution of eccentricity and inclination of 10,000 massless test particles initially spread from 0.5 au to 4 au, with $e=0$, Incl. $=[0-1]^{\circ}$, and $\omega, \Omega, l=[0-360]^{\circ}$ after the $800 \mathrm{kyr}$ instability simulation of Figure 3 . Middle and bottom: evolution of the eccentricity and inclination of Venus (yellow), Earth (blue), and Mars (green) under the effects of the planetary instability shown in Figure 3. Middle: all planets initially with their current orbits. Bottom: all planets initially with circular and planar orbits. Dotted lines in the middle and bottom panels represent the real mean value of eccentricity and inclination for these three planets. The real mean values were evaluated considering an evolution with all planets from Venus to Saturn interacting over a period of $10 \mathrm{Myr}$. The inclination values are referred to the invariable plane (Souami \& Souchay 2012), as defined in the caption of Figure 1.

Mars (green), for both initial configurations, is strongly disturbed, and the terrestrial planets end up with orbits significantly more excited than their present orbits in eccentricity (the real mean values of the present orbits of these planets for both eccentricity and inclination are also shown in Figure 6 middle and bottom panels for comparison). Instead, 
the final orbital inclinations are similar to the real ones, particularly when the terrestrial planets start from their presentday orbits (see Figure 6 middle and bottom panels). Finally, as commonly used to quantify the orbital excitement of the terrestrial planetary system, we considered the angular momentum deficit metrics (AMD; Laskar 1997) in the form presented by Chambers (2001). The current AMD of the terrestrial planets from Mercury to Earth is $\sim 0.0014$. The same value considering only Venus, Earth, and Mars is $\mathrm{AMD}_{\mathrm{VEM}} \sim 0.0009$. As for comparison, because we are not considering Mercury in our simulations, we should use the second value. The AMD for the simulation of the middle panel of Figure 6 is, at the end of the simulation, $\sim 0.0024$ $\left(\sim 2.67 \times \mathrm{AMD}_{\mathrm{VEM}}\right)$, and after taking an average on the orbital $a, e, i$ over the last $200 \mathrm{kyr}$ for all three planets, it is $\sim 0.0018\left(\sim 2.00 \times \mathrm{AMD}_{\mathrm{VEM}}\right)$. Similarly, the AMD for the simulation of the bottom panel of the Figure 6 is, at the end of the simulation, $\sim 0.0023\left(\sim 2.55 \times \mathrm{AMD}_{\mathrm{VEM}}\right)$, and after taking an average on the orbital $a, e, i$ over the last $200 \mathrm{kyr}$ for all three planets, it is $\sim 0.0019\left(\sim 2.11 \times\right.$ AMD $\left._{\text {VEM }}\right)$.

Of course one could also argue that, in the early instability scenario, after the dissipation of the solar gas nebula, the system of planetary embryo precursors of the terrestrial planets could be disturbed in a manner similar to the fully formed terrestrial planets in the case of a late instability. In fact, Clement et al. (2018) found values of AMD two to three times the current value. However, dynamical friction from collisionally generated fragments (not considered in Clement et al. 2018) could potentially damp the final orbits of the terrestrial planets to match the present-day inner solar system (Chambers 2013; Walsh \& Levison 2016). Instead, if the instability happened late, well after terrestrial planet formation, the presence of a remnant planetesimal disk and collisionally generated fragments is unlikely, and therefore the simulations presented here argue that the terrestrial planets would acquire orbits too excited in eccentricities. The same result was presented by Kaib \& Chambers (2016). On the other hand, Roig et al. (2016) obtained good final orbits for terrestrial planets with late giant planet instabilities, while also explaining the excitation of the orbit of Mercury. Therefore, it is important to have in mind that there are some evolutions during the giant planet instability that make this possible, even though the one presented here is not among them.

On this issue, some new constraints should be mentioned. Marty et al. (2017) showed that about $20 \%$ of the $\mathrm{Xe}$ in Earth's atmosphere should have a cometary origin. However, there is no trace of cometary Xe in Earth's interior (Caracausi et al. 2016). This suggests that the cometary bombardment (presumably associated with the giant planet instability) occurred after the formation of the terrestrial crust. Because Earth's formation and mantle crystallization took about 60-100 Myr (Kleine et al. 2009), this would imply that the instability did not occur before this time. Perhaps enough planetesimals (possible collisional debris from debris of the moon-forming event; Bottke et al. 2006) were still present in the terrestrial planet region in the early aftermath of Earth formation (in fact, the Earth accreted about $0.5 \%$ of its mass from them, the so-called Late Veneer) to damp the overexcitation of the terrestrial planets' orbits. This remains to be shown.

\section{Subsequent Evolution of the MB}

We now extend the evolution of the excited MB presented in Figure 2 throughout the age of the solar system and then compare it to the current MB.

For simplicity, we consider the asteroid distribution shown in the Figure 2 right panel as our initial distribution, even though it was obtained in a simulation without terrestrial planets. Following Deienno et al. (2016), we then plugged into the simulation all planets from Venus to Saturn in their current orbits and evolve the $\mathrm{MB}$ of Figure 2 right panel for an additional $\sim 4$ Gyr (with no interpolation, using once again the Mercury package, Chambers 1999, in the hybrid option with a time step of 10 days). Because in the simulation performed to get Figure 2 the final semimajor axes of both Jupiter and Saturn were within $5 \%$ of their present values, and their final eccentricities and inclinations were also close to their present values, with most of the asteroids not in mean motion resonances with them, we do not expect that instantaneously changing the planetary system causes a relevant perturbation in the asteroids' distribution. On the contrary, we believe this to be the best way to continue the evolution over the solar system age, in order to be able to compare the final distribution of the asteroids with the current distribution.

Figure 7 shows a comparison between the orbital $(a, e, i)$ distribution of the $\mathrm{MB}$ resulting from our $4 \mathrm{Gyr}$ extended simulation $^{11}$ (blue) to the present-day $\mathrm{MB}$ (red) for real asteroids with ${ }^{12} H<10$.

It is clear that our simulated MB provides a good match to the real one. Of course there are a few differences. The major difference to note is the number of objects above the $\nu_{6}$ resonance compared to the number of objects below the $\nu_{6}$ resonance for $a<2.5 \mathrm{au}$, which is much larger in our simulated $\mathrm{MB}$ (with a ratio of objects above/below $\sim 1.3$ ) than in the current $\mathrm{MB}(\sim 0.09)$. This is similar to what Deienno et al. (2016) found from their simulations of evolution and reshaping of the GT's MB $(\sim 1.2)$. The ratio we found is nevertheless a lot better than the ones presented in Walsh \& Morbidelli (2011; 5.2, as a result of smooth migration for Jupiter and Saturn) and Clement et al. (2018; 2.24, within JJ instability scenarios that do not take criterion D too seriously). If we exclude the asteroids with inclinations above $20^{\circ}$ at the end of the JJ evolution, the final ratio would be $\sim 0.07$. This suggests that the asteroid inclination excitation in the region within $a<2.5$ au was in reality a bit weaker than in our nominal $\mathrm{JJ}$ simulation, which is plausible given the enormous variety of evolutions that the planets may have potentially had during a $\mathrm{JJ}$ instability phase.

Finally, defining as main belt region the one with $q>1.9$ au and $a<3.2 \mathrm{au}$, we find that $\sim 78 \%$ of the main belt population is lost during the planetary instability. An additional $\sim 43 \%$ was lost within the first $100 \mathrm{Myr}$ after the planetary instability due to the inclusion of the terrestrial planets. Another $\sim 25 \%$ is lost in the subsequent evolution, totaling $\sim 57 \%$ of material loss after the planetary instability. In terms of mass, considering that the current $\mathrm{MB}$ has about $\sim 5 \times 10^{-4} M_{\oplus}$ (DeMeo \& Carry 2013), our predictions suggest that within the original MB region there should be about $\sim 53 \times 10^{-4} M_{\oplus}$, about 10 times more mass than now. Even so, $\sim 53 \times 10^{-4} M_{\oplus}$ is very

\footnotetext{
11 An animation of the entire evolution can be found electronically at http:// staff.on.br/rodney/rogerio/mb-evo.mp4.

12 From http://www.minorplanetcenter.org/iau/MPCORB.html.
} 


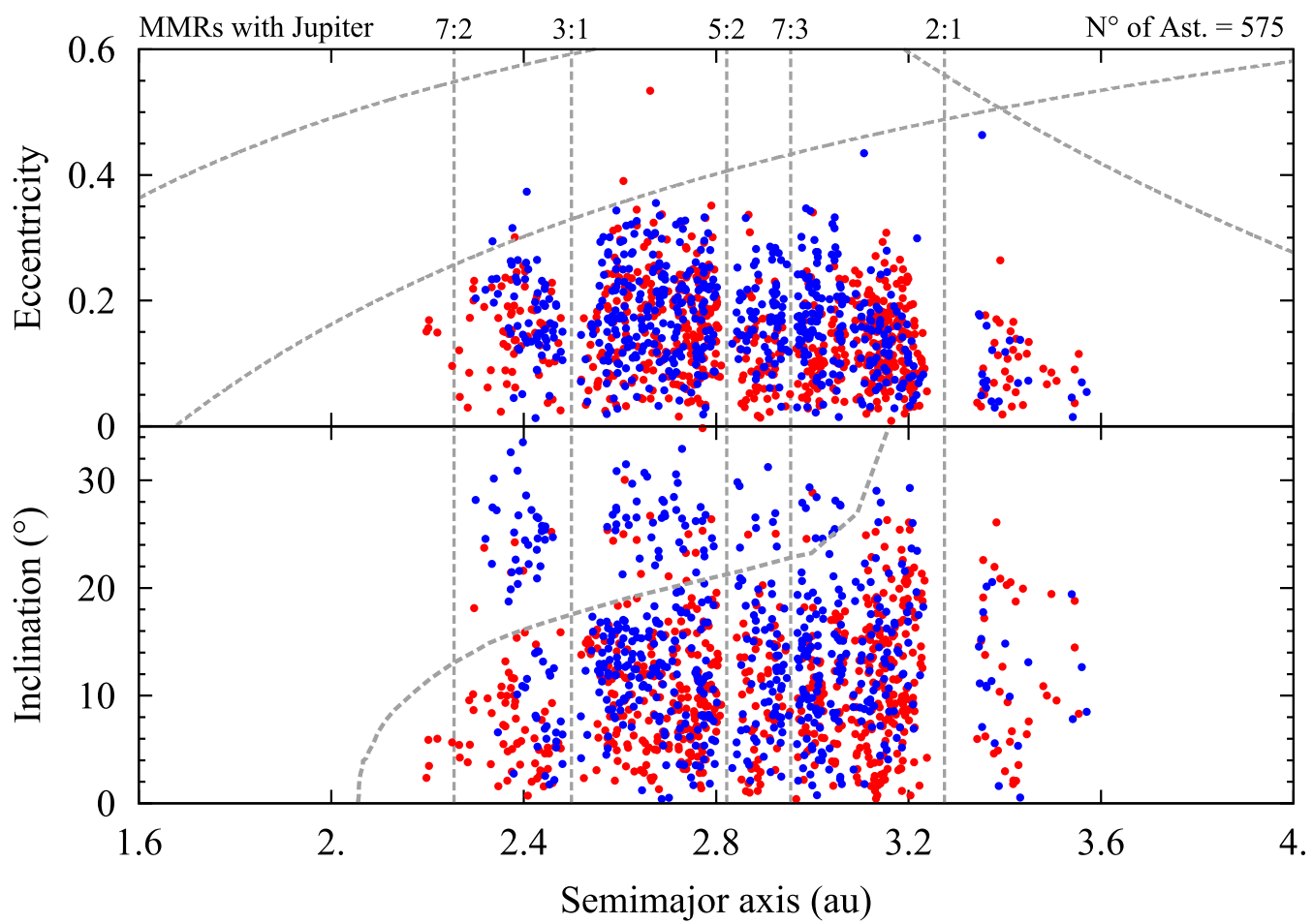

Figure 7. Comparison between the eccentricity (top) and inclination (bottom) as a function of the semimajor axis of the MB resulting from our 4 Gyr extended simulation (blue) to the present-day MB (red) with all $H<10$. The vertical dashed lines show the MMRs between asteroids and Jupiter. The curved dashed lines in the top panel represent the boundaries of the Earth-, Mars-, and Jupiter-crossing orbits, from left to right. The location of the $\nu_{6}$ resonance is also shown in the bottom panel for reference.

little mass, so the scenario proposed in this paper is consistent with the low-mass asteroid belt hypothesis (Levison et al. 2015b; Ogihara et al. 2015; Izidoro et al. 2015, 2016; Moriarty \& Fischer 2015; Dra̧żkowska et al. 2016; Raymond \& Izidoro 2017a, 2017b).

We have shown that a JJ instability produces roughly an order of magnitude mass depletion in the MB. Simulations by Clement et al. (2018) started from a smooth radial surface density distribution and included about an Earth mass in the MB. If we simply applied our depletion factor, the setup in Clement et al. (2018) would produce an MB two orders of magnitude more massive than the present-day one. However, it is worth noting that a second depletion mechanism acts on a more massive belt. Gravitational self-stirring by resident planetary embryos excites and depletes the belt (Chambers \& Wetherill 2001; Petit et al. 2001). Of course, a more massive primordial belt (as modeled by Clement et al. 2018) must undergo much stronger depletion than a primordial low-mass belt (as we have modeled). The simulations of Clement et al. (2018) did not have high-enough resolution to determine the final MB mass; rather, their success criterion was simply that no embryos could survive in the belt (see Figure 1 in Raymond et al. 2009). It remains to be seen whether a combination of self-stirring and an early $\mathrm{JJ}$ instability can provide the requisite depletion.

\section{Conclusion}

We have shown that a JJ evolution during the giant planet instability can excite a dynamically cold primordial main asteroid belt to an overexcited state (comparable to those obtained in both the Grand Tack model, Walsh et al. 2011, and in the chaotic excitation, Izidoro et al. 2016), which subsequently evolves to the current level of excitation due to the preferential removal of the most dynamically excited asteroids over the solar system age.

We started by performing instability simulations of the giant planets from an initial five-planet multiresonant configuration (with resonant period ratios of 3:2, 3:2, 2:1, 3:2; Deienno et al. 2017), as suggested by hydrodynamical simulations of planet migration in a gas-dominated disk. We selected a "nominal" simulation, which satisfies all constraints already considered for the JJ instability scenario (Nesvorný \& Morbidelli 2012; Deienno et al. 2017). Then we restricted our attention to the JJ instability period and recorded for every $1 \mathrm{yr}$ output the orbits of Jupiter, Saturn, and pl5 (the ejected extra ice giant planet predicted by Nesvorný 2011; Batygin et al. 2012; and Nesvorný \& Morbidelli 2012). We interpolated the recorded orbits of the planets to simulate their effect over an initially dynamically cold MB.

We found that Jupiter is dynamically responsible for exciting the entire MB. The other planets, Saturn and pl5, have only minor direct effects upon the MB, but play an important role in the excitation mechanism by making Jupiter acquire a rapidly varying nonnegligible orbital inclination during the instability phase.

The mechanism that excites the MB from an initially cold state to a very excited one is the presence of large and rapidly evolving forced vectors of eccentricity $\left(e_{\text {forced }}\right)$ and inclination $\left(I_{\text {forced }}\right)$ due to the eccentric, inclined, and rapidly changing orbit of Jupiter. Because the secular phases of the asteroids are rapidly randomized, different asteroids achieve different amplitudes of oscillation during the JJ instability phase. Thus, asteroids are spread all over the parameter space of orbital eccentricity and inclination. Once the instability has ended and Jupiter and Saturn reach their present regular orbits, the $\left(e_{\text {forced }}\right)$ 
and $\left(I_{\text {forced }}\right)$ forced vectors reduce in amplitude and evolve regularly. Consequently, the asteroids are frozen with their acquired proper eccentricities and inclinations. Our mechanism is similar to that presented by Izidoro et al. (2016), but in our case we do not require that Jupiter and Saturn remain in the 2:1 MMR for a long time. Our scenario is consistent with Jupiter and Saturn being originally in the 3:2 MMR (Masset \& Snellgrove 2001).

Our results also suggest that the kind of evolution that Jupiter has to have during the $\mathrm{JJ}$ instability to excite the MB is more consistent with an early instability in the solar system rather than a late instability, although new constraints (Marty et al. 2017) indicate that the instability nevertheless postdated terrestrial planet formation. Finally, we showed that the subsequent evolution of the excited MB throughout the age of the solar system makes the final distribution of asteroids quite consistent with the present-day asteroid main belt orbital configuration.

We find that if the asteroid belt had originally comprised a large mass, as assumed in Clement et al. (2018) from the minimum-mass solar nebula model, the giant planet instability alone would not have removed enough mass from the MB region. In this case, an additional depletion mechanism like the Grand Tack or with some temporary embedded embryos within the MB (Raymond et al. 2009; Clement et al. 2018) should be invoked. Still, it remains to be demonstrated whether a combination of the Clement et al. (2018) model with temporary embedded embryos in the MB and self-stirring from a massive disk with an early JJ instability like shown in this work can provide the required depletion. On the other hand, our results support the low-mass asteroid belt model (Izidoro et al. 2015; Dra̧żkowska et al. 2016; Izidoro et al. 2016; Raymond \& Izidoro 2017a, 2017b), which may provide a coherent alternative to the Grand Tack model for the evolution of the inner solar system.

R.D. acknowledges support provided by grant 2014/020135 from the São Paulo Research Foundation (FAPESP) and CAPES. A.I. is thankful for the financial support provided by grants 2016/12686-2 and 2016/19556-7 from the São Paulo Research Foundation (FAPESP) and CAPES. S.N.R. acknowledges the Agence Nationale pour la Recherche via grant ANR-13-BS05-0003-002 (MOJO).

Software: Mercury (Chambers 1999).

\section{ORCID iDs}

Rogerio Deienno (iD https://orcid.org/0000-0001-6730-7857

André Izidoro (iD https://orcid.org/0000-0003-1878-0634

Rodney S. Gomes (iD https://orcid.org/0000-0001-5712-3042

David Nesvorný (ib https://orcid.org/0000-0002-4547-4301

Sean N. Raymond (ib https://orcid.org/0000-0001-8974-0758

\section{References}

Agnor, C. B., \& Lin, D. N. C. 2012, ApJ, 745, 143

Andrews, S. M., Wilner, D. J., Hughes, A. M., Qi, C., \& Dullemond, C. P. 2009, ApJ, 700, 1502

Baruteau, C., Crida, A., Paardekooper, S.-J., et al. 2014, in Protostars and Planets VI, ed. H. Beuther et al. (Tucson, AZ: Univ. Arizona Press), 667

Batygin, K., \& Brown, M. E. 2016, AJ, 151, 22

Batygin, K., Brown, M. E., \& Betts, H. 2012, ApJL, 744, L3

Bitsch, B., Johansen, A., Lambrechts, M., \& Morbidelli, A. 2015, A\&A, 575, A28
Bottke, W. F., Nesvorný, D., Grimm, R. E., Morbidelli, A., \& O’Brien, D. P. 2006, Natur, 439, 821

Bottke, W. F., Vokrouhlický, D., Minton, D., et al. 2012, Natur, 485, 78

Brasil, P. I. O., Roig, F., Nesvorný, D., et al. 2016, Icar, 266, 142

Brasser, R., Matsumura, S., Ida, S., Mojzsis, S. J., \& Werner, S. C. 2016, ApJ, 821,75

Brasser, R., Morbidelli, A., Gomes, R., Tsiganis, K., \& Levison, F. H. 2009, A\&A, 134, 1790

Brasser, R., Walsh, K. J., \& Nesvorný, D. 2013, MNRAS, 433, 3417

Caracausi, A., Avice, G., Bernard, P., Furi, E., \& Marty, B. 2016, in EGU General Assembly Conf. Abstracts, 18, 16723

Chambers, J. E. 1999, MNRAS, 304, 793

Chambers, J. E. 2001, Icar, 152, 205

Chambers, J. E. 2013, Icar, 224, 43

Chambers, J. E., \& Wetherill, G. W. 1998, Icar, 136, 304

Chambers, J. E., \& Wetherill, G. W. 2001, M\&PS, 36, 381

Clement, M. S., Kaib, N. A., Raymond, S. N., \& Walsh, K. J. 2018, Icar, 311,340

D’Angelo, G., \& Marzari, F. 2012, ApJ, 757, 50

Deienno, R., Gomes, R. S., Walsh, K. J., Morbidelli, A., \& Nesvorný, D. 2016, Icar, 272, 114

Deienno, R., Morbidelli, A., Gomes, R. S., \& Nesvorný, D. 2017, AJ, 153, 153

Deienno, R., Nesvorný, D., Vokrouhlický, D., \& Yokoyama, T. 2014, AJ, 148,25

DeMeo, F. E., \& Carry, B. 2013, Icar, 226, 723

DeMeo, F. E., \& Carry, B. 2014, Natur, 505, 629

Drążkowska, J., Alibert, Y., \& Moore, B. 2016, A\&A, 594, A105

Gomes, R., Nesvorný, D., Morbidelli, A., Deienno, R., \& Nogueira, E. 2018 Icar, 306, 319

Gomes, R. S., Tsiganis, K., Morbidelli, A., \& Levison, H. F. 2005, Natur, 435,466

Gradie, J., \& Tedesco, E. 1982, Sci, 216, 1405

Hansen, B. M. S. 2009, ApJ, 703, 1131

Hayashi, C. 1981, PThPS, 70, 35

Izidoro, A., Haghighipour, N., Winter, O. C., \& Tsuchida, M. 2014, ApJ, 782,31

Izidoro, A., Raymond, S. N., Morbidelli, A., \& Winter, O. C. 2015, MNRAS, 453, 3619

Izidoro, A., Raymond, S. N., Pierens, A., et al. 2016, ApJ, 833, 40

Kaib, N. A., \& Chambers, J. E. 2016, MNRAS, 455, 3561

Kleine, T., Touboul, M., Bourdon, B., et al. 2009, GeCoA, 73, 5150

Kley, W., \& Nelson, R. P. 2012, ARA\&A, 50, 211

Lambrechts, M., Johansen, A., \& Morbidelli, A. 2014, A\&A, 572, A35

Laskar, J. 1997, A\&A, 317, L75

Levison, H. F., Kretke, K. A., \& Duncan, M. J. 2015a, Natur, 524, 322

Levison, H. F., Kretke, K. A., Walsh, K. J., \& Bottke, W. F. 2015b, PNAS, 112,14180

Lykawka, P. S., \& Ito, T. 2013, ApJ, 773, 65

Marty, B., Altwegg, K., Balsiger, H., et al. 2017, Sci, 356, 1069

Masset, F., \& Snellgrove, M. 2001, MNRAS, 320, L55

Masset, F. S., \& Papaloizou, J. C. B. 2003, ApJ, 588, 494

Minton, D. A., \& Malhotra, R. 2010, Icar, 207, 744

Minton, D. A., \& Malhotra, R. 2011, ApJ, 732, 53

Morbidelli, A., Bitsch, B., Crida, A., et al. 2016, Icar, 267, 368

Morbidelli, A., Brasser, R., Gomes, R., Levison, H. F., \& Tsiganis, K. 2010, AJ, 140, 1391

Morbidelli, A., \& Crida, A. 2007, Icar, 191, 158

Morbidelli, A., Nesvorny, D., Laurenz, V., et al. 2018, Icar, 305, 262

Morbidelli, A., Tsiganis, K., Crida, A., Levison, F. H., \& Gomes, R. 2007 ApJ, 134, 1790

Moriarty, J., \& Fischer, D. 2015, ApJ, 809, 94

Morishima, R., Stadel, J., \& Moore, B. 2010, Icar, 207, 517

Murray, C. D., \& Dermott, S. F. 1999, in Solar System Dynamics, ed. C. D. Murray \& S. F. McDermott (Cambridge: Cambridge Univ. Press), 274

Nesvorný, D. 2011, ApJL, 742, L22

Nesvorný, D. 2015, AJ, 150, 68

Nesvorný, D., Roig, F., \& Bottke, W. F. 2017, AJ, 153, 103

Nesvorný, D., Vokrouhlický, D., \& Deienno, R. 2014, ApJ, 784, 22

Nesvorný, N., \& Morbidelli, A. 2012, AJ, 144, 117

O'Brien, D. P., Morbidelli, A., \& Bottke, W. F. 2007, Icar, 191, 434

O’Brien, D. P., Morbidelli, A., \& Levison, H. F. 2006, Icar, 184, 39

Ogihara, M., Kobayashi, H., Inutsuka, S.-i., \& Suzuki, T. K. 2015, A\&A, 579, A65

Petit, J.-M., Kavelaars, J. J., Gladman, B. J., et al. 2011, AJ, 142, 131

Petit, J.-M., Morbidelli, A., \& Chambers, J. 2001, Icar, 153, 338

Pierens, A., \& Nelson, R. P. 2008, A\&A, 482, 333 
Pierens, A., \& Raymond, S. N. 2011, A\&A, 533, A131

Pierens, A., Raymond, S. N., Nesvorny, D., \& Morbidelli, A. 2014, ApJL, 795, L11

Raymond, S. N., \& Izidoro, A. 2017a, Icar, 297, 134

Raymond, S. N., \& Izidoro, A. 2017b, SciA, 3, 9

Raymond, S. N., \& Morbidelli, A. 2014, in Proc. IAU Symp. 310, Complex Planetary Systems (Cambridge: Cambridge Univ. Press), 194

Raymond, S. N., O’Brien, D. P., Morbidelli, A., \& Kaib, N. A. 2009, Icar, 203, 644

Raymond, S. N., Quinn, T., \& Lunine, J. I. 2006, Icar, 183, 265

Roig, F., \& Nesvorný, D. 2015, AJ, 150, 186

Roig, F., Nesvorný, D., \& DeSouza, S. R. 2016, ApJL, 820, L30
Souami, D., \& Souchay, J. 2012, A\&A, 543, A133

Toliou, A., Morbidelli, A., \& Tsiganis, K. 2016, A\&A, 592, A72

Volk, K., \& Malhotra, R. 2017, AJ, 154, 62

Walsh, K. J., \& Levison, H. F. 2016, AJ, 152, 68

Walsh, K. J., \& Morbidelli, A. 2011, A\&A, 526, A126

Walsh, K. J., Morbidelli, A., Raymond, S. N., O’Brien, D. P., \& Mandell, A. M. 2011, Natur, 475, 206

Walsh, K. J., Morbidelli, A., Raymond, S. N., O’Brien, D. P., \& Mandell, A. M. 2012, M\&PS, 47, 1941

Wetherill, G. W. 1978, in Protostars and Planets, ed. T. Gehrels \& M. S. Matthews (Tucson, AZ: Univ. Arizona Press), 565

Wetherill, G. W. 1991, LPSC, 22, 1495 